\title{
Retrievable endoscopic stenting for tuberculous oesophagopleural fistula with empyema
}

\author{
Brian YO Chan, MBChB, Canon KO Chan *, FRCS, FHKAM (Surgery) \\ Department of Surgery, Queen Elizabeth Hospital, Jordan, Hong Kong \\ *Corresponding author: cko296@ha.org.hk
}

An earlier version of this paper was presented at Surgical Grand Round Session of upcoming RCSEd/CSHK Conjoint Scientific Congress 2015 held in Hong Kong on 13 September 2015.

Hong Kong Med J 2017;23:89-92

DOI: $10.12809 / \mathrm{hkmj} 154670$

\section{Case report}

A 23-year-old female was admitted for fever, cough, and right pleuritic pain in January 2015. Chest X-ray revealed right pleural effusion. Subsequent computed tomography of the thorax demonstrated necrotising pneumonitis in the right lower lobe, with large right empyema and multiple left lung centrilobular nodules suspicious of tuberculosis (Fig 1a). On admission, haemoglobin level was only $84 \mathrm{~g} / \mathrm{L}$. Oesophagogastroduodenoscopy (OGD) found a linear deep oesophageal ulceration with suspected fistula opening (Fig 1b), biopsy of which yielded Mycobacterium tuberculosis. Gastrografin swallow confirmed contrast leakage at the lower oesophagus with fistulation to the right hemithorax (Fig 1c), suggesting the occurrence of tuberculous oesophagopleural fistula (OPF) and resulting empyema.

Prompt chest drainage and systemic antibiotics were initiated, targeting the polymicrobial and smear positive tuberculous tissue samples. Subsequent therapeutic OGD with endoscopic stenting was performed. The patient was placed in the left lateral decubitus position and sedated. The linear ulcer at

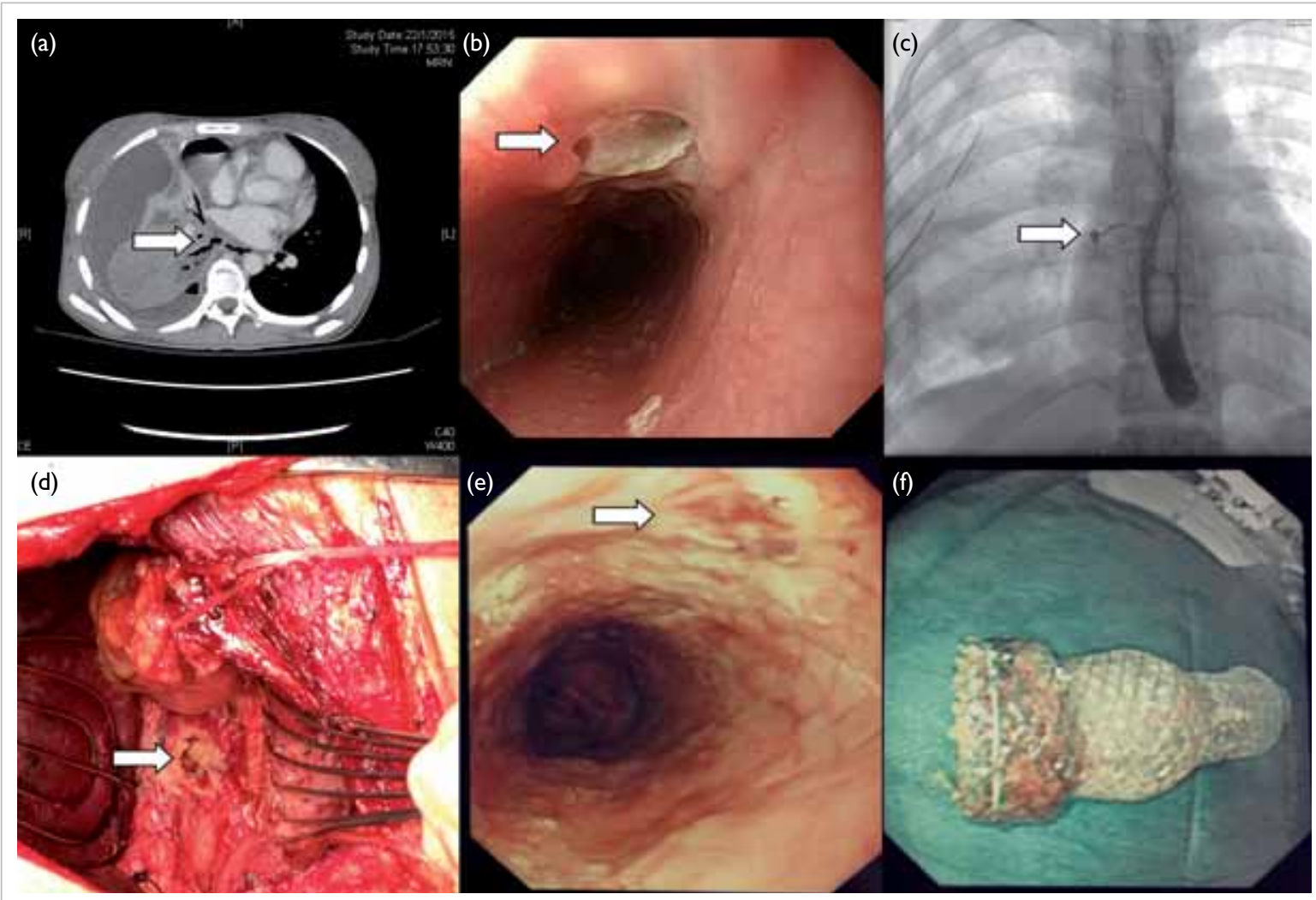

FIG I. Oesophagopleural fistula (arrows)

(a) Computed tomography of the thorax, (b) pretreatment oesophagogastroduodenoscopy, (c) gastrografin swallow, (d) decortication, and (e) complete healing, ( $f$ ) retrieved fully covered self-expandable metallic stent 
24 to $32 \mathrm{~cm}$ from incisor, with $5-\mathrm{mm}$ fistula opening at $24 \mathrm{~cm}$ was visualised. Submucosal lipiodol injection under fluoroscopy marked the margins of the fistulated ulcer, and the most distal end of the linear ulcer (Fig 2a).

A 12-cm 22-Fr retrievable, fully covered, selfexpandable metallic stent (SEMS) with an overthe-wire distal delivery stent deployment system (Taewoong Medical, South Korea) was selected. The shoulders of the stent were placed across the fistula opening, being deployed over the guidewire (Fig $2 b$ and 2c). All positions were confirmed by fluoroscopy and the proximal stent secured with two endoclips at 12 and 6 o'clock position.

The patient tolerated the procedure well. She was put on total parenteral nutrition for 2 weeks, then resumed enteral feeding via a nasoduodenal tube inserted during a separate OGD session. Decortication was later performed to clear the empyema cavity (Fig 1d). Follow-up OGD at 8 weeks demonstrated no stent migration and stent was removed uneventfully by pulling on the purse-string suture with endoscopic forceps. Check endoscopy confirmed complete healing of the ulcer by scarring (Fig 1e). The retrieved fully covered SEMS was intact and without tissue ingrowth (Fig 1f).

Recovery of the patient was satisfactory. She achieved complete resolution of sepsis and tolerated an oral diet after stent removal. Follow-up computed tomography of the thorax in May 2015 showed minimal thoracic collection and no lung consolidation.

\section{Discussion}

Oesophageal stenting has significantly matured since its inception. In unresectable malignant obstruction, SEMS has become the standard of care, with high technical success (83\%-100\%) in positioning and effective dysphagia palliation (80\%-95\%). ${ }^{1}$ Emerging roles are also seen in benign conditions like anastomotic leaks or perforations, with promising $81.4 \%$ and $86 \%$ success in defect closure, respectively. ${ }^{2}$ Success is nonetheless variable in treating benign strictures and refractory oesophageal variceal bleeding. ${ }^{1}$ In malignant oesophagorespiratory fistulae (ORF), 80\% of cases achieve complete healing with SEMS. ${ }^{3}$ Little is known about the efficacy in benign ORF, OPF in particular, due to the clinical rarity and inherent heterogeneity.

Oesophagorespiratory fistulae is an uncommon entity comprising all pathological connections between the oesophagus and airway. Malignant oesophageal, lung, or mediastinal tumours are the most common causes. Fistulation occurs secondary to tumour invasion or inflammation post-radiotherapy, laser, chemotherapy or stenting. Benign ORF is rare, with major causes being trauma and infection. ${ }^{4}$ Regarding fistula subtypes,

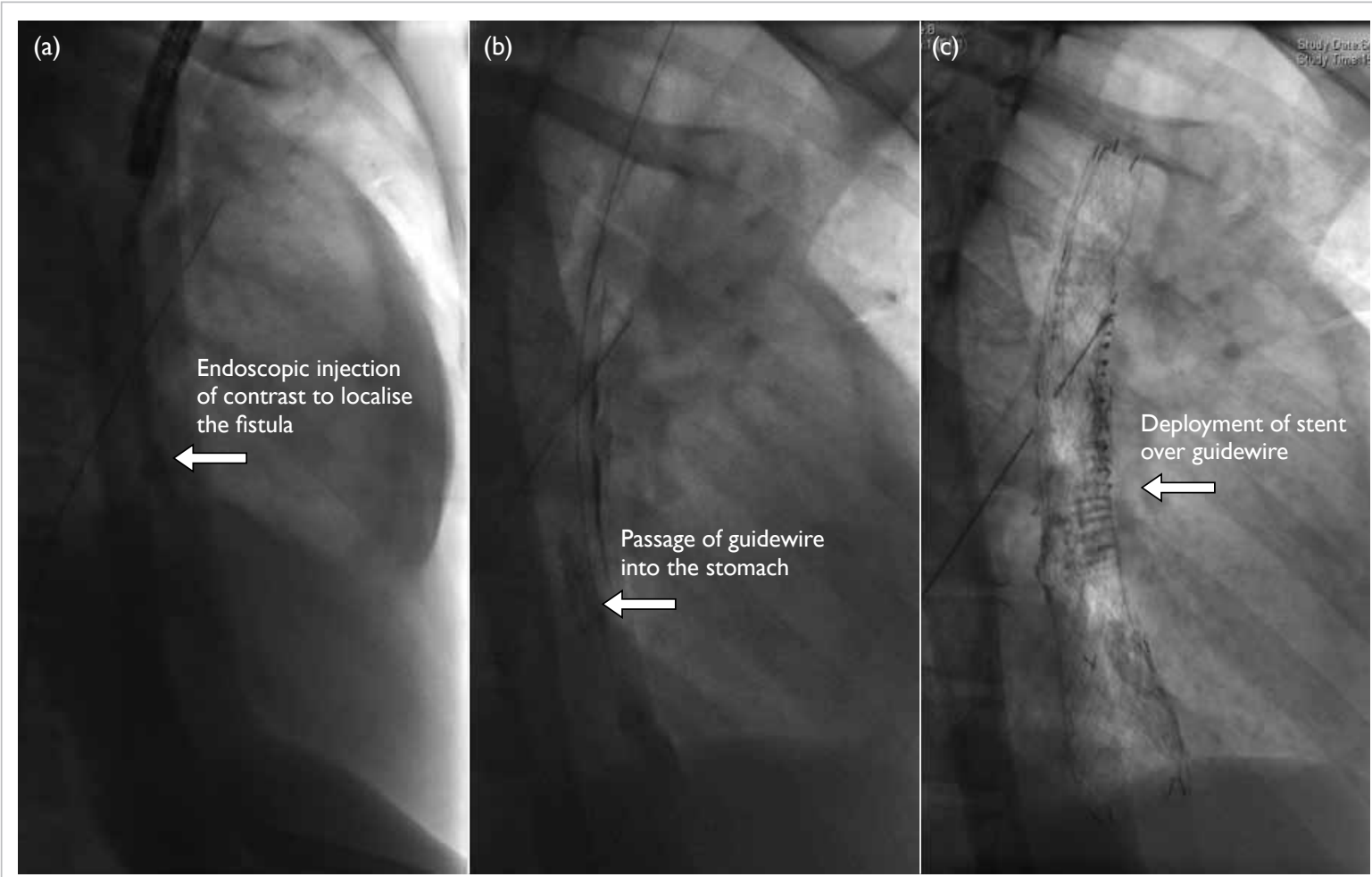

FIG 2. Endoscopic insertion of self-expandable metallic stent

(a) Margin marking, (b) guidewire passage, and (c) stent deployment 
only $3 \%$ to $11 \%$ represent oesophagopulmonary fistula or OPF that communicate peripherally. The rest are either oesophagotracheal (52\%-57\%) or oesophagobronchial $(37 \%-40 \%)$ in location. ${ }^{5}$ It is easy to understand why few reports exist of OPF and its treatment.

In general, OPF has a similar aetiology to ORF. Malignancies aside, it is a well-recognised complication in $0.2 \%$ to $1 \%$ of pneumonectomies. Other traumatic causes include endoscopic sclerotherapy, gunshot wound, and foreign body ingestion. ${ }^{5}$ Infective causes range from tuberculosis to mycotic disease, also candidiasis in immunocompromised individuals. Other reported cases include Crohn's disease, perforated Barrett's ulcer, and ruptured diverticulum.$^{6-8}$ Like postsurgical leaks and perforations, OPF is a sinister condition associated with high morbidity and mortality.,3 Significant mediastinal and pleural contamination, polymicrobial sepsis and abscesses often ensue, warranting multiple drainage procedures and surgery, as well as intensive life support.

Oesophagopleural fistula rarely heals spontaneously. Principles of management include drainage of collection, exclusion of fistulation, aggressive antimicrobials, and lastly, intensive nutritional and organ support. Established abscesses persist despite spillage control by stenting, hence necessitating drainage procedures. ${ }^{9}$ Pleural drainage is possible via tube thoracostomy or image-guided pigtail catheter insertion. Yet if multiloculated collections exist, open or thoracoscopic drainage is preferred since it permits septa breakdown under direct vision, thorough irrigation, and placement of a drain in an ideal position. ${ }^{10}$ Early use of broadspectrum intravenous antibiotics with aerobic and anaerobic coverage is equally important. Fistula exclusion may be operative, fluoroscopic, or endoscopic. Traditional surgical approaches involve either primary suturing or segmental oesophagectomy, but are complex and associated with high morbidity (30\%-50\%) and mortality (10\%). ${ }^{11}$ Bypass and muscle flap buttressing are newer operations possible for the debilitated. ${ }^{12,13}$ Embolisation with vascular plug and coils under fluoroscopy has been reported in one article. Endoscopic fibrin glue application represents a viable option in small-calibre fistulae. Limited reports also exist for other endoscopic measures like argon plasma or electrocoagulation, suturing, or clipping devices. ${ }^{11}$

Our patient represented a promising candidate for oesophageal stenting. Initially performed with rigid plastic tubes, development has evolved to SEMS with easier insertion and less migration. Most commonly made of nitinol, SEMS exhibits great flexibility and radial force to maintain patency and position. They come uncovered, partially, or fully covered, with a plastic or silicon membrane. Fully covered SEMS is the stent-of-choice in managing fistulae, since the covering is vital for leakage prevention and easy retrievability. ${ }^{1}$ Most published studies suggest stent placement immediately after leakage confirmation to minimise mediastinal contamination. ${ }^{3}$ Insertion is via upper endoscopy under sedation, with the patient in a left lateral or prone position to minimise aspiration. Stent size is chosen according to oesophageal diameter and defect size. The proximal and distal ends of the lesion are identified endoscopically, marked by radiopaque dye, then a guidewire advanced over the defect, stent positioned across and deployed overthe-wire, all under endoscopic and fluoroscopic guidance. Potential stent shrinkage or migration is counteracted by allowing 4-6 cm proximal and distal coverage. ${ }^{1,10}$ A feeding tube is routinely inserted for prompt enteral nutrition. No data are available for optimal timing of when to resume an enteral diet, but in oesophageal leaks, Rajan et $\mathrm{al}^{10}$ demonstrated fitness on day 2 following stent placement. Oral intake is not encouraged though due to potential peristalsis-inducing stent migration and hence leakage. No conclusive timing for stent removal exists, with studies in general reporting intervals of 4 to 6 weeks or 6 to 8 weeks. ${ }^{10}$ In our case, stent removal at 8 weeks was without difficulty, with no tissue ingrowth or stent disintegration.

Only one review of SEMS placement in oesophageal fistulae exists. It is reported that $64.7 \%$ clinical success was achieved in defect closure, which is significantly lower than the over $80 \%$ rates in malignant ORF, perforations, and post-surgical leaks.,3 Detailed scrutiny of the seven studies pooled, however, revealed that aortoesophageal and oesophagoatrial fistulae were also included. ${ }^{13}$ These conditions present with life-threatening torrential haemorrhage, and are undoubtedly different in terms of pathogenesis and survival to the majority of ORF where sepsis is the main issue. Moreover, fistulae subtype or location was mostly not reported, and if present, mostly oesophagotracheal. Significant heterogeneity exists. Only one of 24 fistulae was identified as OPF. It is questionable how applicable the data are.

Specific literature is lacking. Our case report aside, Kang et $\mathrm{al}^{4}$ represent the only article reporting endoscopic SEMS for OPF as far as we are aware. Successful technical and clinical outcomes were achieved in both studies. To take this further, considering the similarities in terms of pleural or mediastinal sepsis and treatment goals in minimising further contamination, we believe it is reasonable to extrapolate the success in managing perforations and postsurgical leaks to ORF.

Potential SEMS-related complications include retrosternal pain, oesophageal perforation, 
bronchoesophageal fistulae, stent migration, obstruction, or fracture. Migration of the stent contributes to clinical failure, and appears to be a particularly troubling issue for fully covered SEMS, with $10 \%$ to $25 \%$ occurrence in covered stents and $2 \%$ to $5 \%$ in uncovered. ${ }^{1}$ It is also more frequent in placements for fistulae (33.3\%) compared with benign strictures (15.8\%). ${ }^{14}$ Appropriate stent size and length reduce the risk of migration, balancing the larger radial force to oppose migration against the risk of pressure necrosis. To counteract migration, some stents come with flared proximal ends. ${ }^{3}$ Endoscopic clip application of the proximal stent end to the oesophageal wall has also been reported to be an effective strategy. ${ }^{15}$ Such technological advances may further improve outcomes.

Our case represents the first in local medical literature to report SEMS use in benign OPF. With the global trend towards minimally invasive therapies, we expect a paradigm shift towards effective novel techniques such as endoscopic stenting or repair. In this patient with tuberculous fistulae, we favoured stenting over any endoscopic suturing or clipping to avoid the tension exhibited in inflamed tissue.

\section{References}

1. Kang HW, Kim SG. Upper gastrointestinal stent insertion in malignant and benign disorders. Clin Endosc 2015;48:18793.

2. van Halsema EE, van Hooft JE. Clinical outcomes of selfexpandable stent placement for benign esophageal diseases a pooled analysis of the literature. World J Gastrointest Endosc 2015;7:135-53.

3. Mangiavillano B, Pagano N, Arena M, et al. Role of stenting in gastrointestinal benign and malignant diseases. World J Gastrointest Endosc 2015;7:460-80.

4. Kang GH, Yoon BY, Kim BH, et al. A case of spontaneous esophagopleural fistula successfully treated by endoscopic stent insertion. Clin Endosc 2013;46:91-4.

5. Shin JH, Kim JH, Song HY. Interventional management of esophagorespiratory fistula. Korean J Radiol 2010;11:133-
40.

6. Albuquerque A, Ramalho R, Macedo G. Multiple esophagopleural and esophagobronchial fistulas in a patient with Crohn's disease. Endoscopy 2012;44 Suppl 2:E114-5.

7. Andersson R, Nilsson S. Perforated Barrett's ulcer with esophago-pleural fistula. A case report. Acta Chir Scand 1985;151:495-6.

8. Agarwal V, Singh SK, Siddiqi MS, Joshi LM, Tandon S. Esophagopleural fistula following spontaneous rupture of traction diverticulum. Asian Cardiovasc Thorac Ann 2003;11:344-5

9. Kim KR, Shin JH, Song HY, et al. Palliative treatment of malignant esophagopulmonary fistulas with covered expandable metallic stents. AJR Am J Roentgenol 2009;193:W278-82.

10. Rajan PS, Bansal S, Balaji NS, et al. Role of endoscopic stents and selective minimal access drainage in oesophageal leaks: feasibility and outcome. Surg Endosc 2014;28:236873

11. Koo JH, Park KB, Choo SW, Kim K, Do YS. Embolization of postsurgical esophagopleural fistula with AMPLATZER vascular plug, coils, and Histoacryl glue. J Vasc Interv Radiol 2010;21:1905-10.

12. Kim JJ, Park JK, Moon SW, Park K. A new surgical technique for spontaneous esophagopleural fistula after pneumonectomy: cervical esophagogastrostomy via presternal and subcutaneous route, using a thoracic esophageal mucosal stripping. Thorac Cardiovasc Surg 2013;61:496-8.

13. David EA, Kim MP, Blackmon SH. Esophageal salvage with removable covered self-expanding metal stents in the setting of intrathoracic esophageal leakage. Am J Surg 2011;202:796-801.

14. Buscaglia JM, Ho S, Sethi A, et al. Fully covered selfexpandable metal stents for benign esophageal disease: a multicenter retrospective case series of 31 patients. Gastrointest Endosc 2011;74:207-11.

15. Park SY, Park CH, Cho SB, et al. The usefulness of clip application in preventing migration of self-expandable metal stents in patients with malignant gastrointestinal obstruction [in Korean]. Korean J Gastroenterol 2007;49:49. 